\title{
Aquecimento de materiais estratificados com laser pulsado de Nd:YAG
}

\author{
O. O. Diniz Neto ${ }^{(*)}$
}

\begin{abstract}
Resumo Em nosso trabalho examinamos o aquecimento de amostras compostas de duas e três camadas, condutor-isolante e condutor-condutor, com um pulso potente de laser. O comportamento transiente da distribuição de temperatura através da amostra foi calculado. Em nosso modelo de calculo para o processo de aquecimento levamos em conta a dependência com a temperatura dos parâmetros térmicos (difusividade, capacidade e condutividade térmica), óticos (refletividade e coeficiente de absorção), bem como a dependência das condições de contorno com o tempo e consequentemente com a temperatura. Aplicamos nossa metodologia para calcular o aquecimento amostras compostas: Al$\mathrm{Au}, \mathrm{Al}-\mathrm{Ag}, \mathrm{Al}-\mathrm{Al}_{2} \mathrm{O}_{3}, \mathrm{Al}-\mathrm{Au}-\mathrm{Ag}$ e Ag-Au-Al. Concluimos que o substrato influencia as temperaturas máximas nas superfície exposta ao laser e a forma com que a frente de calor se propaga nas amostras termicamente finas.
\end{abstract}

Palabras clave: Multicapas. Láser pulsado Nd: YAG. Tratamiento térmico. Perfil térmico.

\section{Heating of multi-layered samples by a Nd:YAG pulsed laser}

\begin{abstract}
In the work we examine the heating of multi-layered samples by a powerful $\mathrm{Nd}-\mathrm{YAG}$ pulsed laser. The samples are made of two and three layers, conductor-isolator $\left(\mathrm{Al}-\mathrm{Al}_{2} \mathrm{O}_{3}\right)$, conductor-conductor $(\mathrm{Al}-\mathrm{Ag} ; \mathrm{Al}-\mathrm{Au})$ and conductor-conductor-conductor $(\mathrm{Al}-\mathrm{Au}-\mathrm{Ag} ; \mathrm{Ag}-\mathrm{Au}-\mathrm{Al})$. The transient behaviour of the temperature distribution throughout the sample is computed. We carry out three dimensional model calculations for the heating process in which we consider not only the temperature dependence of the sample thermal and optical parameters but also the space and time characteristics of the laser beam as the heating source. We showed the influence of the substrate in the thermal profile, in space and time, and maximum temperature on the multi - layered samples.
\end{abstract}

Keywords: Multilayers. Nd:YAG pulsed laser. Heat treatment. Thermal profile.

\section{INTRODUÇÃO}

Com a sofisticação crescente de diversas áreas cientificas e tecnológicas, há a necessidade de um estudo detalhado de como a radiação laser interage com materiais estratificados. Necessidade que fica evidente ao analisarmos os diversos casos que existem na literatura científica de amostras de varias camadas de materiais que são irradiados com laser como por exemplo: discos de gravação ótica (1-3) que possuem quatro camadas, (substrato, camada absorvedora do laser, camada de gravação, camada protetora); aquecimento e fusão de filmes finos sobre substratos isolantes (4); implantação de ions e impurezas sobre semicondutores (5); crescimento de cristais $(5,6)$; recozimento de semicondutores

(*) Dep. Ciência Física, Univ. Federal de Uberlândia, Minas Gerais (Brasil). sobre isolantes (7) e tratamento térmico de superfícies metálicas (8-10). O número crescente de aplicações tecnológicas importantes envolvendo a interação de lasers com amostras estratificadas, o nosso desejo de investigar a fundo a possibilidade de um controle paramétrico sobre a frente de calor a medida que o fluxo de calor se propaga na amostra, e o estudo das influencias dos substratos nas temperaturas alcançadas pelas amostras, motivou-nos a desenvolver um algoritmo que resolvesse a equação de difusão de calor para este caso. Nosso algoritimo leva em conta a dependência com a temperatura dos parâmetros térmicos (difusividade, capacidade e condutividade térmica), óticos (refletividade e coeficiente de absorção), bem como a dependência das condições de contorno com o tempo e consequentemente com a temperatura. Aplicamos nosso modelo em amostras compostas de duas e três camadas, condutor-isolante e condutor-condutor ( $\mathrm{Al}-\mathrm{Au}, \mathrm{Al}$ - 
$\mathrm{Ag}, \mathrm{Al}-\mathrm{Al}_{2} \mathrm{O}_{3}, \mathrm{Al}-\mathrm{Au}-\mathrm{Ag}$ e $\mathrm{Ag}-\mathrm{Au}-\mathrm{Al}$ ), aquecidas com um pulso potente de laser. O comportamento transiente da distribuição de temperatura através da amostra foi calculado.

\section{MODELO FÍSICO-MATEMÁTICO}

Consideramos em nossa formulação, para o processo de aquecimento de um sólido exposto a um feixe de laser, a dependência dos parâmetros que regem o aquecimento com a temperatura. Assim, para descrevermos a difusão de calor pela amostra, temos que resolver uma equação diferencial parabólica não-linear para cada camada do sólido (equação (1)). Admitiremos que na interface das camadas há continuidade do fluxo de calor (equação (2)) e que a resistência térmica entre as camadas é desprezível (equação (3)). Supondo que as amostras estão no vácuo, não há perda de calor por condução ou convecção, havendo perdas apenas por radiação. Assim, as condições de contorno levam em conta somente a radiação de calor pelas superfície externas das amostras.

Fisicamente, o problema que consideramos consiste em obter o mapeamento tridimensional do comportamento transiente da temperatura induzida pelo laser através do sólido. As amostras são cilíndricas e obedecem a lei de Beer para absorção ótica. O pulso do laser possui perfil temporal gaussiano ( $\tau$ é a duração do pulso)e a distribuição espacial também gaussiano ( $R_{\mathrm{o}}$ é largura do pulso). A geometria do pulso laser e da amostra apresentam uma clara simetria circular, como mostra a figura [1a]. Usaremos, obviamente, coordenadas cilíndricas em nossa formulação. $\mathrm{O}$ laser incide sobre o ponto $z=$ 0 na superfície da amostra. A fração de energia incidente do pulso que converte em calor, após a absorção pela amostra, depende da temperatura, pois os parâmetros óticos refletividade $R(T)$ e coeficiente de absorção $\alpha(T)$ variam com a temperatura. Como conseqüência, a temperatura da amostra $T(\pi$, $z, t)$ é determinada por uma fonte de calor volumétrica $A(\pi, z, t, T)$ que varia espacialmente e temporamente com o aquecimento da amostra. E ainda, buscando um modelo mais completo, consideramos que os parâmetros térmicos que controlam a difusão de calor para o interior da amostra variam com a temperatura. O problema torna-se completamente não linear, cuja solução analítica ainda não é conhecida. Para resolve-lo buscamos soluções numéricas baseadas em esquemas auto-consistentes.

Para implementarmos nosso modelo numérico, partimos da equação não linear parabólica de difusão de calor em cada camada da amostra:

$$
\begin{gathered}
C_{m}(T) \frac{\partial T_{m}}{\partial t}= \\
=\nabla \cdot\left(K_{m}(T) \nabla T_{m}\right)+A(\stackrel{r}{r}, t, T)
\end{gathered}
$$

onde $m$ indexa a camada e os parâmetros relevantes em nosso modelo, que são: $C_{m}(T)$, capacidade térmica por unidade de volume $\left(\mathrm{J} \mathrm{cm}^{-3} \mathrm{~K}^{-1}\right) ; K(T)$, condutividade térmica $\left(W \mathrm{~cm}^{-1} K^{-1}\right) ; \kappa(T)$, difusivi-
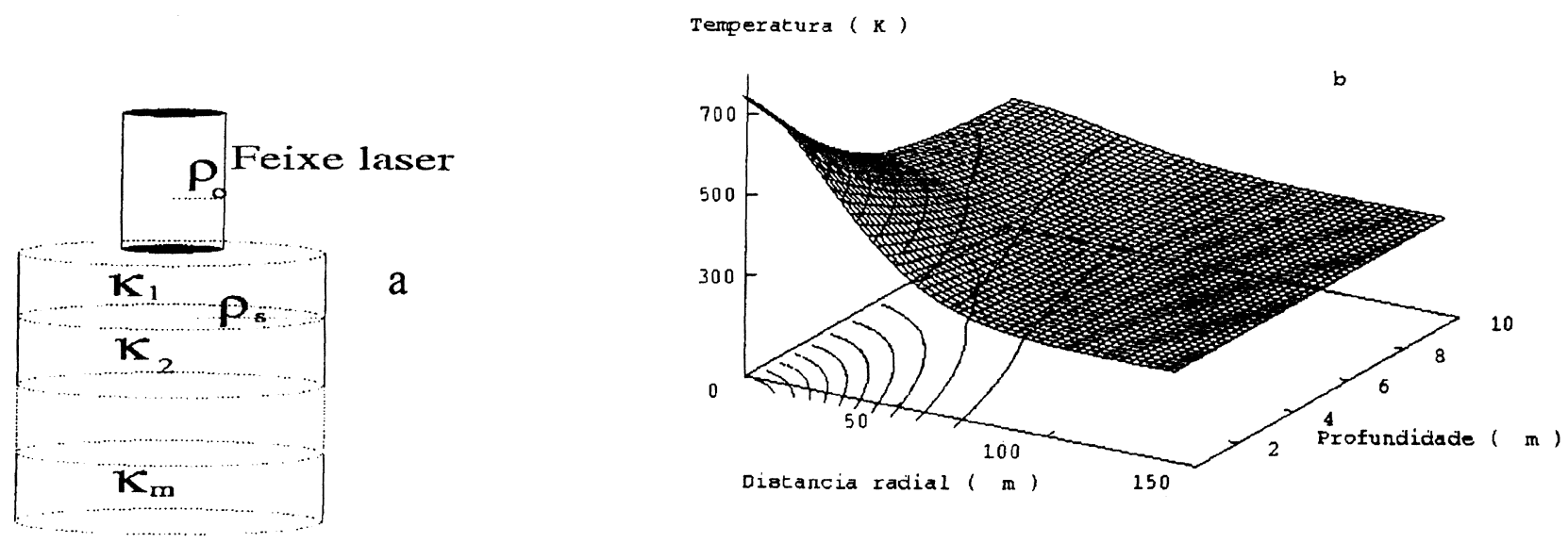

FIG. 1.- Amostra de múltiplas camadas com laser com simetria circular fig. [1a]. Distribuição espacial de temperatura, no instante $t=1,8 \tau_{0}$ para amostra de Al-Ag (fig. [1b]) de $10 \mu \mathrm{m}$ de espessura, $150 \mu \mathrm{m}$ de raio, aquecida com um pulso laser gaussiano com $\tau_{0}=250 \mathrm{~ns}, \mathrm{I}_{0}=30 \mathrm{MW} / \mathrm{cm}^{2} \mathrm{e} \pi_{0}=50 \mu \mathrm{m}$.

FIG. 1.- The geometry assumed in the model calculations, multi-layered samples and pulsed laser with circular simmetry [1a]. Temperature space distribution, in the time $t=1,8 \tau_{o}$ for an $L=10 \mu \mathrm{m}$ thick and $150 \mu \mathrm{m}$ of radius sample of Al-Ag [1b]. Irradiation settings: $N d: Y A G$ Gaussian pulse, $\tau_{o}=250 \mathrm{~ns}, I_{o}=30 \mathrm{MW} / \mathrm{cm}^{2}$ and $\pi_{0}=50 \mu \mathrm{m}$. 
dade térmica $\left(\mathrm{cm}^{2} \mathrm{~s}^{-1}\right) ; \alpha K(T)$, coeficiente de absorção $\left(\mathrm{cm}^{-1}\right) ; R(T)$, refletividade. A fonte volumétrica de aquecimento possui simetria circular e sua forma geral em coordenadas cilíndricas é:

$$
\begin{gathered}
A(\stackrel{\perp}{r}, t, T)= \\
=I_{0}[1-R(T)] \alpha(T) e^{-\left[\alpha(T) z-\mid\left(\frac{\rho}{\rho 0}\right)^{2}-\eta\left(\frac{t}{\tau-1.5}\right)^{2}\right.}
\end{gathered}
$$

A equação (2) descreve um pulso de laser gaussiano, onde, $\eta$ é uma constante de normalização, calculada de tal forma que a integral de $A(\vec{r}, t, T)$ no volume da amostra e ao longo da duração total do pulso de irradiação, corresponde à energia absorvida pela amostra; $\rho_{0}$ é a largura do feixe; $\tau_{0}$ (FWHM) do pulso. A refletividade $R(T)$ e o coeficiente de absorção $\alpha(T)$ variam com a temperatura. A temperatura $T(\rho, z, t)$ será, é claro, independente da coordenada polar angular.

As condições de contorno nas superfícies externas das amostras são:

$$
\begin{gathered}
K(T)=\frac{\partial T(\rho, z, t)}{\partial z}=\varphi(T)-\phi_{z=0}(T) \\
K(T)=\frac{\partial T(\rho, z, t)}{\partial z}=\phi_{z=L}(T) \\
K(T)=\frac{\partial T(\rho, z, t)}{\partial z}=\phi_{z=\rho_{s}}(T)
\end{gathered}
$$

onde $\varphi(r, 0, t, T)$ é a intensidade do laser absorvida pela amostra e $\phi(r, L, t, T)=\varepsilon \sigma\left(T^{4}-T_{0}{ }^{4}\right)$ é o fluxo de energia irradiada pela superfície da amostra de emissividade $\varepsilon$ e $\sigma$ é a constante de Stefan-Boltzmann.

Nas interfaces das camadas admite-se que há continuidade do fluxo de calor e que a resistência térmica entre as camadas é desprezível, então obtém-se:

$$
\begin{aligned}
K_{m} \frac{\partial T}{\partial z} & =K_{m+1} \frac{\partial T_{m+1}}{\partial z} \\
T_{m} & =T_{m+1}
\end{aligned}
$$

A equação diferencial (1) é não- linear e o primeiro passo para resolve-la é a aplicação da transformada de Kirchhoff (12), que introduz uma nova variável denominada de temperatura não-linear ' $\theta$ ' através de:

$$
\theta(\stackrel{r}{r}, t)=\frac{1}{K_{m_{0}}} \int_{T_{0}}^{T} K_{m}\left(T^{\prime}\right) d T^{\prime}
$$

A equação transformada em coordenadas cilíndricas adquire a forma:

$$
\begin{gathered}
\frac{1}{\kappa(T)} \cdot \frac{\partial \theta}{\partial t}=\frac{1}{\rho} \cdot \frac{\partial}{\partial \rho}\left(\rho \frac{\partial \theta}{\partial \rho}\right)+ \\
+\frac{\partial^{2} \theta}{\partial z^{2}}+\frac{A(\stackrel{r}{r}, t, T)}{K_{0}}
\end{gathered}
$$

Analisando a equação (7) sujeita as condições de contorno (3), (4), (5) e ao termo de fonte(2), dependentes da temperatura, observamos que ela é fortemente não linear requerendo uma solução numérica para sua resolução. A metodologia de tratamento que demos ao problema envolve a solução numérica da equação não linear de calor. Desenvolvemos e exploramos um novo algoritmo, específico para tratar a formulação dada a questão pelo presente trabalho. Nele, a discretização das funções e derivadas que aparecem na equação de difusão é feita através do método das diferenças finitas. Usamos uma versão modificada, que desenvolvemos (1), da formulação de Crank-Nicholson para obtermos um sistema de equações algébricas acopladas, que foi resolvido pelo método iterativo das Sobre Relaxações Sucessivas (SOR).

\section{RESULTADOS E DISCUSSÕES}

Nestas secção, apresentaremos aplicações do método para investigar o aquecimento a laser de materiais estratificados compostos de duas ou três camadas. Em todos os cálculos apresentados nesta secção, tomaremos para o pulso laser $\rho_{0}=50 \mu \mathrm{m}$, $\tau_{0}=250 \mathrm{~ns}$ e $I_{0}=30 \mathrm{MW} / \mathrm{cm}^{2}$. As amostras consideradas são cilíndricas com espessura $L=10 \mu \mathrm{m}$, e raio $\rho_{i}=150 \mu \mathrm{m}$. Sob a hipótese de que as amostras estão no vácuo, as condições de contorno só levaram em conta as perdas de calor por radiação térmica, que é proporcional a $T^{4}$. Uma questão sempre relevante nos processos de aquecimento a laser é a natureza do substrato. Isto tem particular relevância para o caso de filmes finos suportados que devam ser processados a laser, bem como, em espelhos usados para conduzir um feixe de laser intenso. Para investigar como o substrato afeta as temperaturas na superfície de uma amostras termicamente fina, estudamos sistematicamente o caso de amostras em que a primeira camada era de alumínio, com $5 \mu \mathrm{m}$ de espessura, e a segunda camada foi considerada como contendo um dos seguintes materiais: $\mathrm{Ag}, \mathrm{Au}$ e $\mathrm{Al}_{2} \mathrm{O}_{3}$. Estes materiais tem diferentes parâmetros térmicos e foram dados acima em ordem decrescente de difusividade térmica. As expressões de como os parâmetros físicos, para o $\mathrm{Au}$, 

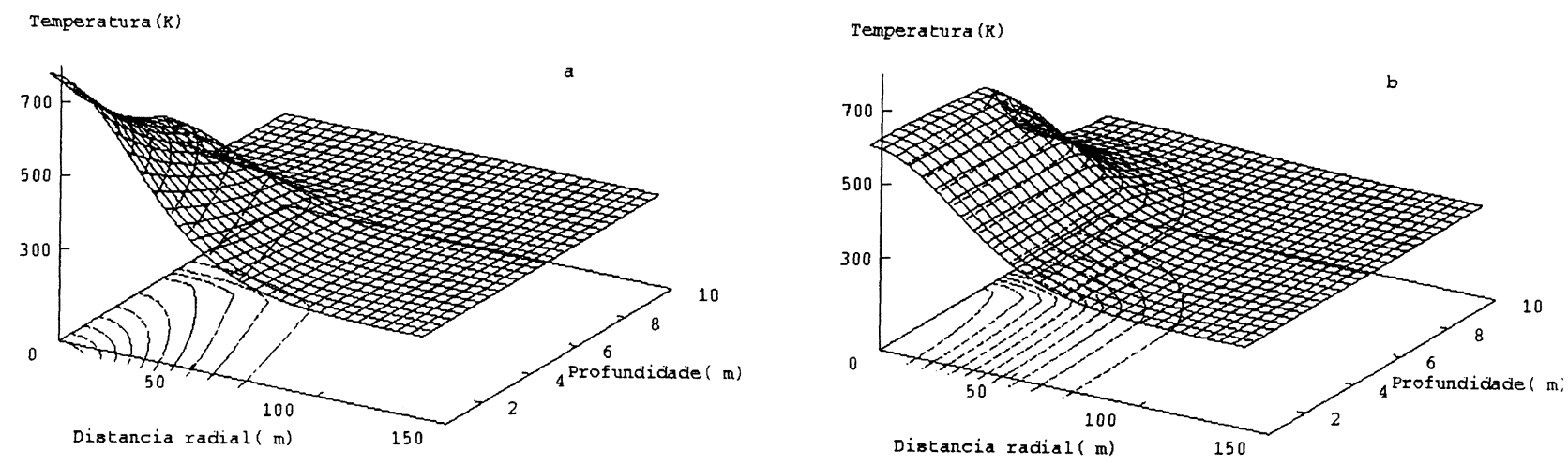

FIG. 2.- Distribuição espacial de temperatura, nos instantes $t=1,8 \tau_{0}$ [fig.2a] e $t=3,6 \tau_{0}$ [fig.2b], para a amostra de $\mathrm{Al}-\mathrm{Al}_{2} \mathrm{O}_{3}$ de $10 \mu \mathrm{m}$ de espessura, $150 \mu \mathrm{m}$ de raio, aquecida com um pulso laser gaussiano com $\tau_{0}=250 \mathrm{~ns}, \mathrm{I}_{0}=30 \mathrm{MW} / \mathrm{cm}^{2}$ e $\pi_{0}=50 \mu \mathrm{m}$

FIG. 2.- Temperature space distribution, in the times $t=1,8 \tau_{o}[2 a]$ and $t=3,6 \tau_{o}$ [2b] for an $L=10 \mu \mathrm{m}$ thick and $150 \mu \mathrm{m}$ of radius sample of $\mathrm{Al}_{-} \mathrm{Al}_{2} \mathrm{O}_{3}$. Irradiation settings: $\mathrm{Nd}: Y A G$ Gaussian pulse, $\tau_{o}=250 \mathrm{~ns}$, $I_{o}=30 \mathrm{MW} / \mathrm{cm}^{2}$ and $\pi_{0}=50 \mu \mathrm{m}$.

$\mathrm{Ag}$ e $\mathrm{Al}$, variam com a temperatura, usadas neste trabalho são as mesma utilizadas por $\mathrm{O}$. O. Diniz Neto et al. (11). Para $\mathrm{o} \mathrm{Al}_{2} \mathrm{O}_{3}$ temos que a condutividade térmica e a difusividade térmica variam com a temperatura sugundo as expressões: 95,63 $\mathrm{T}^{-1,2556} ; 519,08 \mathrm{~T}^{-1,2711}$ respectivamente.

Apresentamos, neste trabalho, as distribuições tridimensionais de temperatura e os perfis de temperatura com a profundidade nos tempos $t=1,8 \tau_{0}$, que corresponde ao momento em que a temperatura no centro da amostra atinge um valor máximo, e $t=$ $1,8 \tau_{0}$, em que praticamente não ocorre variação de temperatura no centro da amostra, com a profundidade para a camada de $\mathrm{Al}$ na amostra de $\mathrm{Al}-\mathrm{Al}_{2} \mathrm{O}_{3}$. Os resultados dos cálculos com nosso modelo estão nas figuras [2a] e [2b], onde mostramos os perfis tridimensionais de temperatura e as isotermas correspondentes para a amostras $\mathrm{Al}-\mathrm{Al}_{2} \mathrm{O}_{3}$ em $t=1,8 \tau_{0}$ e $t=3,6 \tau_{0}$, respectivamente. Os resultados para as distribuições tridimensionais de temperatura para a amostra $\mathrm{Al}-\mathrm{Au}$ e Al-Ag em $t=1,8 \tau_{0}$ estão na figura [1b]. As figuras [3a], [3b] mostram os perfis de profundidade térmicos axiais em $t=1,8 \tau_{0}$ e $t=3,6 \tau_{0}$, respectivamente, para todas as amostras. Para comparação incluímos, também, o perfil correspondente para o caso de uma amostra homogênea de $\mathrm{Al}$ de $10 \mu \mathrm{m}$ de espessura. Conclusões interessantes, e de grande valor prático para o processamento de materiais com lasers, emanam da análise detalhada dos resultados acima apresentados. Nota-se, por exemplo, a profunda diferença entre as isotermas no caso em que o substrato é um bom condutor e no caso em que ele é mau condutor de calor. Quando, por exemplo, o substrato é metálico, a distribuição de temperatura acompanha ó perfil espacial do pulso laser. Se, ao contrário, o substrato é isolante térmico, como $\mathrm{Al}_{2} \mathrm{O}_{3}$, o perfil é significativamente modificado. Outra conclusão importante emerge da inspeção da figura [3]: quanto maior a difusividade térmica do substrato menor a temperatura da superfície exposta ao laser e maior a temperatura da superfície oposta (base do substrato), o que se da em conseqüência da maior facilidade da difusão de calor pelo substrato. É digno de registro a extrema sensibilidade do nosso algoritmo de cálculo permitindo que variações, mesmo que pequenas, nos parâmetros controladores do processo global de aquecimento a laser, se traduzam em variações concretas, ainda que pequenas, na temperatura do sólido em pontos localizados quer na superfície exposta ao laser, quer no corpo da amostra e mesmo na sua face oposta. Isso ocorre, mesmo quando a diferença entre as difusividade térmicas dos componentes é pequena, como para o caso do ouro e da prata. Veja, por exemplo, que as temperaturas máximas atingidas são $798 \mathrm{~K}, 768 \mathrm{~K}$, $765 \mathrm{~K}$ e $763 \mathrm{~K}$ na superfície, enquanto que em pontos simetricamente opostos, na base, os resultados

Isso ocorre, mesmo quando a diferença entre as difusividade térmicas dos componentes é pequena, como para o caso do ouro e da prata. Veja, por exemplo, que as temperaturas máximas atingidas são $798 \mathrm{~K}, 768 \mathrm{~K}, 765 \mathrm{~K}$ e $763 \mathrm{~K}$ na superfície, enquanto que em pontos simetricamente opostos, na base, os resultados correspondentes são $300 \mathrm{~K}$, $365 \mathrm{~K}, 371 \mathrm{~K}$ e $380 \mathrm{~K}$ respectivamente para as amostras de $\mathrm{Al}-\mathrm{Al}_{2} \mathrm{O}_{3}, \mathrm{Al}, \mathrm{Al}-\mathrm{Au}$ e $\mathrm{Al}-\mathrm{Ag}$.

Enfim, como mais uma extensão de nossa metodologia de cálculo, aplicamo-lo em amostras de três camadas. Na primeira amostra arranjamos os mate- 

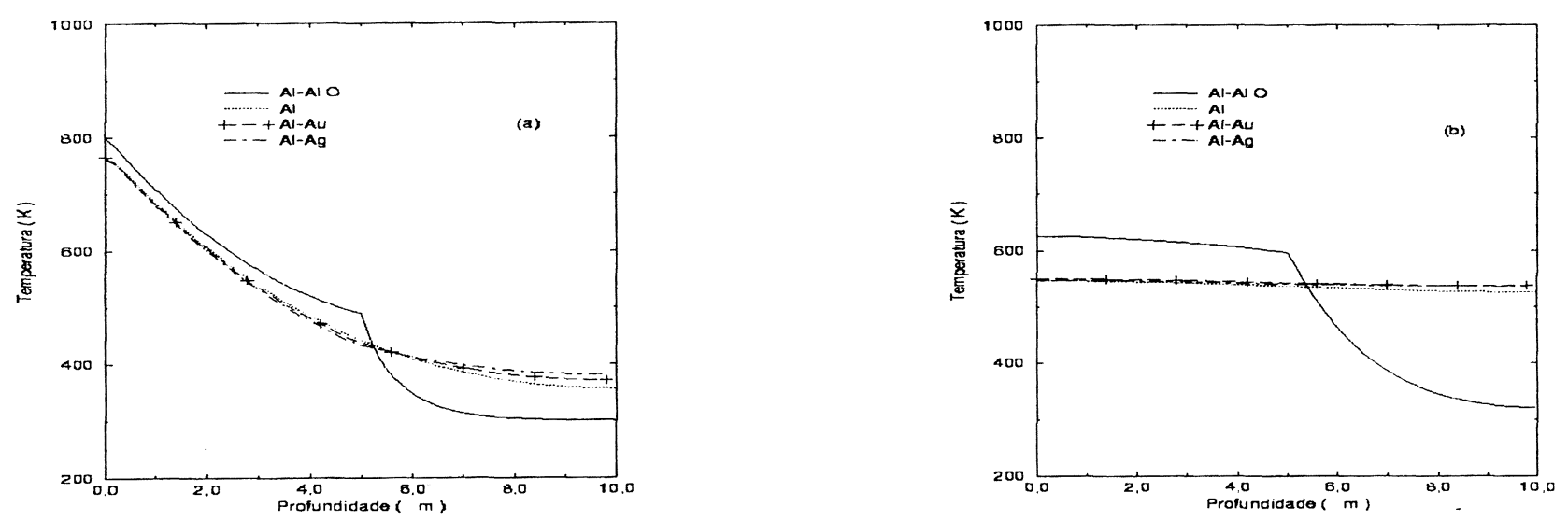

FIG. 3.- Temperatura nos centros das amostras, nos instantes $t=1,8 \tau_{0}$ [fig.3a] e $t=3,6 \tau_{0}$ [fig. 3b], para amostras de $\mathrm{Al}-\mathrm{Al}_{2} \mathrm{O}_{3}, \mathrm{Al}, \mathrm{Al}-\mathrm{Au}$ e $\mathrm{Al}-\mathrm{Ag}$ de $10 \mu \mathrm{m}$ de espessura, $150 \mu \mathrm{m}$ de raio, aquecida com um pulso laser gaussiano com $\tau_{0}=250 \mathrm{~ns}, \mathrm{I}_{0}=30 \mathrm{MW} / \mathrm{cm}^{2}$ e $\pi_{0}=50 \mu \mathrm{m}$.

FIG. 3.-Axial temperature profile, in the times $t=1,8 \tau_{o}[3 a]$ and $t=3,6 \tau_{o}[3 b]$ for an $L=10 \mu \mathrm{m}$ thick and $150 \mu \mathrm{m}$ of radius samples of $\mathrm{Al}_{-} \mathrm{Al}_{2} \mathrm{O}_{3}, \mathrm{Al}, \mathrm{Al}-\mathrm{Au}$ and Al-Ag. Irradiation settings: Nd:YAG Gaussian pulse, $\tau_{o}=250 \mathrm{~ns}, I_{o}=30 \mathrm{MW} / \mathrm{cm}^{2}$ and $\pi_{0}=50 \mu \mathrm{m}$.

riais em ordem crescente de difusividade térmica, a saber, Al-Au-Ag. Na segunda, vamos colocá-los em ordem oposta Ag-Au-Al. As resistências térmicas nas interfaces serão desprezadas e as temperaturas nas faces de contato serão iguais. Os resultados de nossos cálculos, sob a forma da distribuição espacial de temperatura no instante em que o centro da superfície da amostra alcança a temperatura máxima e as isotermas correspondentes estão na figu$\mathrm{ra}[4 \mathrm{a}]$. A evolução temporal dos perfis de profundi- dade térmicos axiais é mostrada na figura[4b], para as amostra $\mathrm{Ag}-\mathrm{Au}-\mathrm{Al}$ e $\mathrm{Al}-\mathrm{Au}-\mathrm{Ag}$.

\section{CONCLUSÃO}

Neste trabalho, comprovamos a importância do substrato na determinação da distribuição espacial da temperatura em amostras termicamente finas. Estudando sistematicamente o efeito do substrato
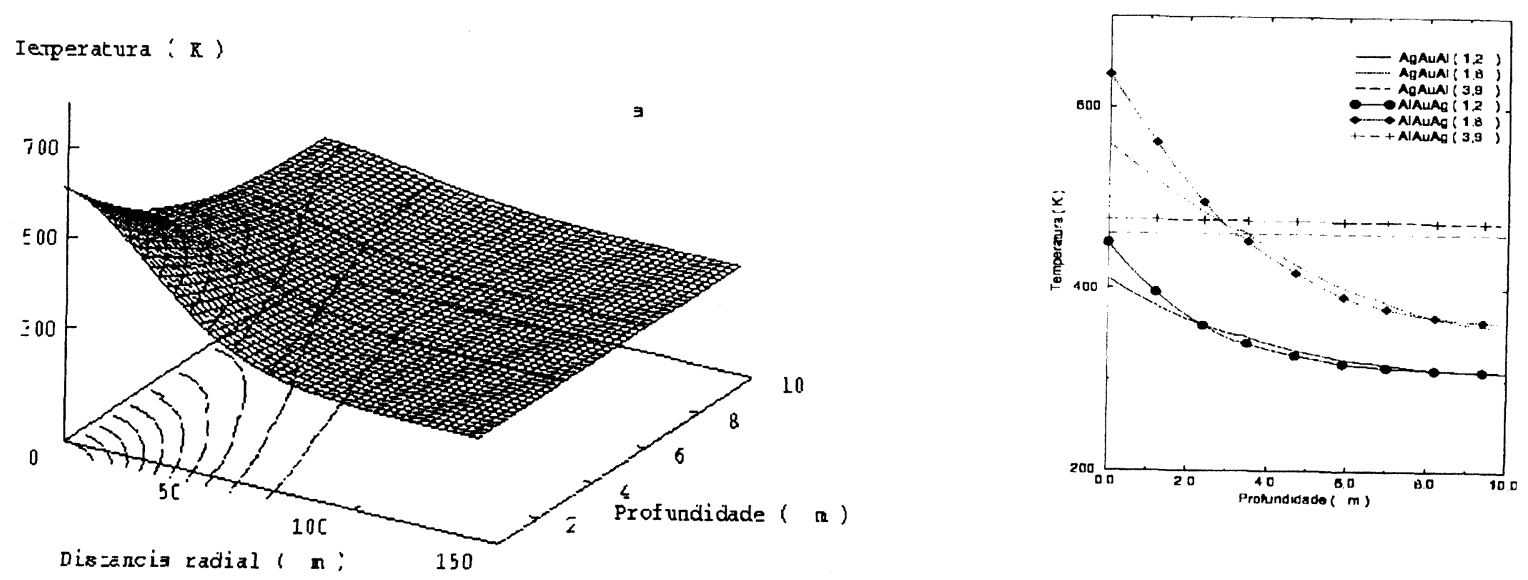

FIG. 4.- Distribuição espacial de temperatura no instante $t=1,8 \tau_{0}$ para amostra de Al-Au-Ag [fig. 4a]; e evolução temporal das temperaturas axiais em função da profundidade para amostras de Ag-Au-Al e Al-AuAg [fig.4b] de $10 \mu \mathrm{m}$ de espessura, $150 \mu \mathrm{m}$ de raio, aquecida com um pulso laser com $\tau_{0}=250 \mathrm{~ns}$, $\mathrm{I}_{0}=30 \mathrm{MW} / \mathrm{cm}^{2}$ e $\pi_{0}=50 \mu \mathrm{m}$.

FIG. 4.- Temperature space distribution in the times $t=1,8 \tau_{o}$ of sample Al-Au-Ag [4a]; time sequence of the axial temperature profile, in the times $t=1,2 \tau_{o} ; 1,8 \tau_{o}$ and 3,9 $\tau_{o}$ for an $L=10 \mu \mathrm{m}$ thick and $150 \mu \mathrm{m}$ of radius samples of Al-Au-Ag and Ag-Au-Al [4b]. Irradiation settings: $N d$ : YAG Gaussian pulse, $\tau_{o}=250 \mathrm{~ns}$,

$$
I_{o}=30 \mathrm{MW} / \mathrm{cm}^{2} \text { and } \pi_{0}=50 \mu \mathrm{m} \text {. }
$$


sobre as temperaturas nas superfícies das amostras, conseguimos evidenciar pequenas variações de temperaturas, ainda que os parâmetros térmicos dos substratos se modificassem muito pouco, como no caso de uma amostra de alumínio com substrato de ouro ou de prata. Ficou claro que quanto menor forem as condutividades e as difusividades térmicas dos substratos, maiores serão as temperaturas atingidas na superfície da amostra. As formas das isotermas dependem também, significativamente dos substratos, como podemos comprovar comparando as isotermas das amostras de alumínio com substrato bom condutor de calor com as amostras com substrato mau condutor de calor. Enquanto no primeiro caso as isotermas, em primeira aproximação, acompanham o perfil do pulso laser, no segundo caso, quando o substrato é $\mathrm{Al}_{2} \mathrm{O}_{3}$, as isotermas sofrem profundas modificações. Observamos o papel desempenhado pelos parâmetros óticos, analisando as amostras compostas de três camadas e verificamos que a amostra $\mathrm{Ag}-\mathrm{Au}-\mathrm{Al}$ possui temperaturas menores do que a amostra $\mathrm{Al}-\mathrm{Au}-\mathrm{Ag}$ devido ao fato da refletividade da prata ser maior que a do alumínio. Finalmente, concluímos que possuímos um modelo físico-matemático eficiente para estudar o aquecimento de materiais estratificados com laser.

\section{Agradecimento}

Agradecemos a FAPEMIG pelo apoio financeiro, sem o qual este trabalho não poderia ser realizado.

\section{REFERENCIAS}

(1) ANDERSON, R.J. 64 (12), 1988: 6639-6645.

(2) Koyanagi, H., Ito, T., Miyamoto, N., Soto, Y.J. Appl. Phys. 66 (3), 1989: 1045-50.

(3) Madison, M.R., McDeniel, T.W. J. Appl. Phys. 66 (12), 1989: 5738-5748.

(4) Imen, K., Lin, J.Y. e Allen, S.D. J. Appl. Phys. 66 (2), 1989: 488-491.

(5) Burgener, M.L. e Reedy, R.E. J. Appl. Phys. 53 (6), 1982: 4357-4363.

(6) Calder, I.D. e Sue, E. J. Appl. Phys. 53 (11), 1982: 7545-7550.

(7) Waechter, D., Schvan, P., Thomas, R.E. J. Appl. Phys. 59 (10), 1986: 3371-3374.

(8) Vilar, R., Colaço, R. e Almeida, A. Opt. Quantum Electronics 27, 1994: 1273-1289.

(9) GutiérRez, A., DamboreneA, J. Appl. Phys. A 63 1996: $461-465$

(10) Tsunemi, A. et. al. Appl. Phys. A 63 1996: 435-439.

(11) Diniz Neto, O.O. e Lima, C.A.S. J. Phys. D: Appl. Phys. 27, 1994: 1795-1804. 